\title{
The RSc0454-Encoded FAD-Linked Oxidase Is Indispensable for Pathogenicity in Ralstonia solanacearum GMI1000
}

\author{
Xun Hu, Zhiwen Zhao, Tao Zhuo, Xiaojing Fan, and Huasong Zou ${ }^{\dagger}$ \\ State Key Laboratory of Ecological Pest Control for Fujian and Taiwan Crops, College of Plant Protection, Fujian Agriculture \\ and Forestry University, Fuzhou 350002, China
}

Accepted 10 December 2018.

\begin{abstract}
Ralstonia solanacearum is the causal agent of bacterial wilt disease. Here, we report that a large FAD-linked oxidase encoded by RSc0454 in GMI1000 is required for pathogenicity. The FADlinked oxidase encoded by $\mathrm{RSc} 0454$ is composed of 1,345 amino acids, including DUF3683, lactate dehydrogenase (LDH), and succinate dehydrogenase (SDH) domains. The RSc0454 protein showed both LDH and SDH activities. To investigate its role in pathogenicity, a deletion mutant of the RSc0454 gene was constructed in GMI1000, which was impaired in its ability to cause bacterial wilt disease in tomato. A single DUF3683, LDH, or SDH domain was insufficient to restore bacterial pathogenicity. Mutagenesis of the RSc0454 gene did not affect growth rate but caused cell aggregation at the bottom of the liquid nutrient medium, which was reversed by exogenous applications of lactate, fumarate, pyruvate, and succinate. qRT-PCR and promoter LacZ fusion experiments demonstrated that RSc0454 gene transcription was induced by lactate and fumarate (both substrates of LDH). Compared with the downregulation of the succinate dehydrogenase gene $s d h B A D C$ and the lactate dehydrogenase gene $l d h$, RSc0454 gene transcription was enhanced in planta. This suggests that the oxidase encoded by RSc0454 was involved in a redox balance, which is in line with the different living conditions of $R$. solanacearum.
\end{abstract}

An electron transport chain is composed of a series of complexes that mediate both reduction and oxidation reactions to transfer electrons from donors to acceptors (Milenkovic et al. 2017). In eukaryotes, electron transport chains are located in the inner mitochondrial membrane and in the thylakoid membrane of the chloroplast in photosynthetic eukaryotes (Alric and Johnson 2017). In complex I, the NADH coenzyme Q reductase catalyzes the oxidation of Krebs-cycle electron carrier NADH and passes these electrons on to coenzyme Q. Complex II (succinate dehydrogenase $[\mathrm{SDH}]$ ) is involved in an alternative pathway that supplies electrons to coenzyme Q. The cytochrome

${ }^{\dagger}$ Corresponding author: H. Zou; hszou@ fafu.edu.cn

Funding: This work was supported by National Natural Science Foundation of China grants (31671988 and 31872919) and a Fujian Provincial Department of Science and Technology grant (the Guiding Project of Fujian Province/2016N0006).

*The $\boldsymbol{e}$-Xtra logo stands for "electronic extra" and indicates that two supplementary figures and two supplementary tables are published online.

(c) 2019 The American Phytopathological Society bc1 complex (complex III) accepts electrons from coenzyme Q and then passes these to complex IV (cytochrome c oxidase). Complex IV is the final step in electron transfer, which produces water via reduction of molecular oxygen (Acin-Perez and Enriquez 2014). In bacteria, the electron transport chain is found in the cell plasma membrane, with a variety of electron donors and acceptors (Thauer et al. 1977). When organic matter is supplied as the energy source, the donor may be either NADH or succinate, and the electrons enter the electron transport chain via NADH dehydrogenase (complex I) or SDH (complex II) (Heikal et al. 2014; Tran et al. 2006). Other dehydrogenases include lactate dehydrogenase (LDH), glyceraldehyde-3-phosphate dehydrogenase, and formate dehydrogenase (Thauer et al. 1977). Most dehydrogenases show increased expression levels in bacterial cells if metabolic requirements are triggered by the environment.

$\mathrm{LDH}$ catalyzes the interconversion of pyruvate to lactate under anaerobic growth conditions (Lodi et al. 1994). In addition to mammalian, plant, and yeast mitochondria (Passarella et al. 2014), D-LDH activity has been extensively reported in a range of microbes (Bunch et al. 1997; Gao et al. 2015; Garvie 1980). Deletion of LDH in Bacillus cereus leads to a slow growth rate, as well as a reduced expression of fermentative genes (Laouami et al. 2011). SDH is the only dehydrogenase that participates in both the electron transport chain and in the citric acid cycle. This dehydrogenase is composed of the four subunits SDHA, SDHB, SDHC, and SDHD (Janssen et al. 1997). SDHA (FAD-containing flavoprotein) and SDHB (iron-sulfur protein) are two hydrophilic parts that form the catalytic core of the complex, conferring SDH activity (Grimm 2013). So far, very few studies have reported the requirement for $\mathrm{LDH}$ and $\mathrm{SDH}$ in the pathogenicity of plant pathogens. The mitochondrial D-LDH MoDLD1 is involved in the maintenance of redox homeostasis during conidial germination and contributes to the virulence of Magnaporthe oryzae (Zhou et al. 2017). Silencing SDH in Phytophthora sojae causes significantly reduced virulence and the production of shorter and more bifurcated hyphae (Pan et al. 2017).

Ralstonia solanacearum is a soilborne pathogen that causes bacterial wilt disease in more than 250 plant species. Due to its wide distribution and severe economic loss, bacterial wilt has been ranked second among the top 10 most destructive bacterial plant diseases (Mansfield et al. 2012). Wounded roots or natural openings are preferred invasion pathways for $R$. solanacearum, before it enters into the host xylem through the intercellular space of the plant root cortex and the vascular parenchyma (Leonard et al. 2017). R. solanacearum utilizes several mechanisms to colonize host plants throughout the infection. At an early invasion stage, $R$. solanacearum efficiently translocates toward wound saps or plant exudates with the aid of chemotaxis 
and aerotaxis (Yao and Allen 2006, 2007). Since it is necessary for chemotaxis, motility plays an important role in bacterial pathogenicity, and nonmotile mutants with defects in either fliC or flhDC are unable to move toward host roots (Tans-Kersten et al. 2001). At this point, both mutants have shown remarkably reduced virulence in soil-soak inoculation assays; however, they exhibit virulence similar to the wild-type strain when directly inoculated into the plant stem (Tans-Kersten et al. 2004; Yao and Allen 2006). In addition, $R$. solanacearum has evolved multiple redundant reactive oxygen species (ROS)-scavenging enzymes to cope with the intense oxidative stress caused by host plants (Flores-Cruz and Allen 2009). Inside the host xylem, $R$. solanacearum consumes the available $\mathrm{O}_{2}$ in the xylem fluid, thus lowering the $\mathrm{O}_{2}$ content (Dalsing et al. 2015). Since xylem sap contains extremely low levels of organic acids, amino acids, and carbon resources, $R$. solanacearum must adapt to a nutrient- and oxygen-limited environment (Fatima and Senthil-Kumar 2015).

In this work, we report the function of a large FAD-linked oxidase encoded by RSc0454 in $R$. solanacearum GMI1000. This oxidase has both LDH and SDH activities and can only be found in restricted soilborne microbes. In addition to the requirement for pathogenicity, this oxidase seemed to be involved in the redox balance through coordination with the standard LDH and SDH encoded by $l d h$ and $s d h B A D C$ genes. Our results indicate that a complex redox mechanism is essential for $R$. solanacearum pathogenicity.

\section{RESULTS}

\section{Structure and distribution}

of FAD-linked oxidase RSc0454 from $R$. solanacearum.

In the genome sequence of $R$. solanacearum GMI1000, $\mathrm{RSc0454}$ is annotated as a FAD-linked oxidase gene. Its nucleotide sequence is $4,035 \mathrm{bp}$ and encodes a large protein of 1,345 amino acids. Based on the conserved domain research in GenBank, the RSc0454 protein has been found to have three distinctive independent domains. The first 144 amino acids at the $\mathrm{N}$-terminus are the conserved domain of DUF3683 and are typically found in microbes with unknown function. Position 160 to position 819 contains a FAD-containing D-LDH domain. Position 862 to position 1,309 contains a $4 \mathrm{Fe}-4 \mathrm{~S}$ dicluster domain combined with a SDH iron-sulfur subunit (Fig. 1A). The conformed structure domains were further validated through 3D modeling prediction in RaptorX (Fig. 1B), and the predicted second structure contains $44 \% \alpha$-helix, $10 \% \beta$-sheet, and $44 \%$ coil structures.

A sequence homology search in GenBank showed that the $\mathrm{RSc0454}$ protein was only found in species of Ralstonia, Pandoraea, Cupriavidus, Paraburkholderia, and Burkholderia. Within the genus Ralstonia, the homologies among different species shared more than $94 \%$ amino acid sequence identity. The RSc0454 protein from GMI1000 showed 84-87\% identity with the homologies of Cupriavidus species and showed $80-82 \%$ identity with the homologies of Pandoraea, Paraburkholderia, and Burkholderia. Eighteen homologies from representative species were selected for phylogenetic relationship analysis. Five sequences from Ralstonia species were clustered into one group. Homologies of Cupriavidus species showed a close relationship with the RSc0454 protein. In comparison, the homologies of Paraburkholderia ginsengiterrae, $P$. phenoliruptrix, and $P$. rhynchosiae were far removed from those of the Ralstonia group (Fig. 1C).

The RSc0454 protein has both LDH and SDH activities.

The specific structure composition suggested to investigate whether the RSc0454 protein has both LDH and SDH activities. Several mutation sites were always found in the PCR product when the entire 4,035-bp coding sequence of the RSc0454 gene was amplified. Therefore, the coding sequence of RSc0454 was divided into two parts and sequentially cloned into the vector

A

\begin{tabular}{|rlrrr|r}
1 & & 819 & 862 & 1309 & 1345 \\
\hline DUF3683 & LDH domain & & & SDH domain \\
\hline
\end{tabular}

B

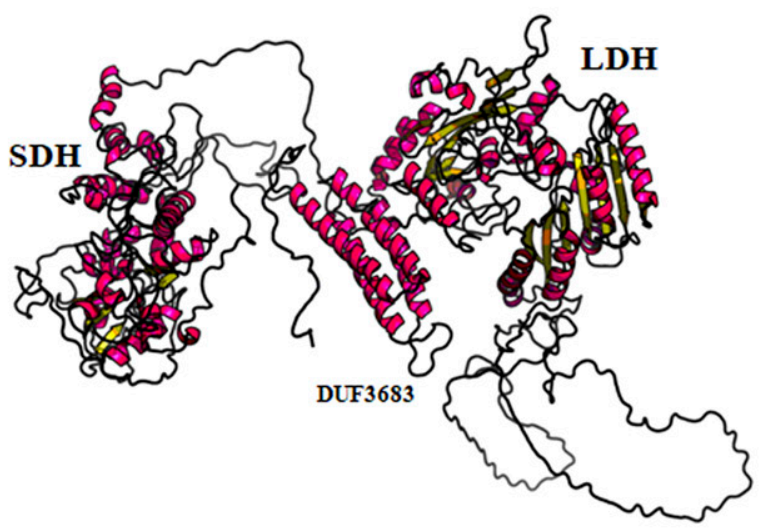

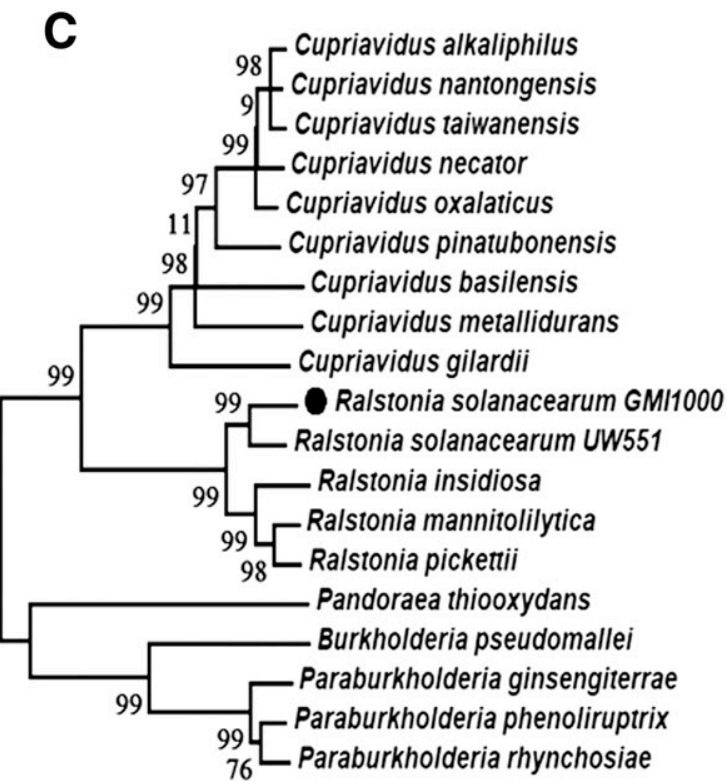

$\stackrel{\overbrace 0 2}{0.02}$

Fig. 1. Sequence analysis of the large FAD-linked oxidase encoded by RSc0454 in Ralstonia solanacearum. A, Schematic diagram of the RSc0454 protein structure. The conserved DUF3683, lactate dehydrogenase (LDH), and succinate dehydrogenase (SDH) domains were obtained by a BLASTP search in the GenBank database. B, 3D shape of the RSc0454 protein predicted with RaptorX. The analysis was performed in structure prediction mode based on the amino acid sequence. C, Evolutionary relationships of RSc0454 from $R$. solanacearum GMI1000 with its 18 homologies in other microbes. The evolutionary history was inferred using the neighbor-joining method in MEGA5. The confidence probability (multiplied by 100) for an interior branch length above 0 was estimated using the bootstrap test (1,000 replicates), and the results are shown next to the branches. The tree is drawn to scale, with branch lengths provided in the same units as those of the evolutionary distances used to infer the phylogenetic tree. The location of RSc0454 in R. solanacearum GMI1000 is indicated with a black dot. 
pMD19-T (simple). Next, the entire coding sequence was cloned into pET41a (+) (Supplementary Fig. S1). After induction and sonication, part of the $\mathrm{RSc} 0454$ protein was found to be absorbed by cell debris. To eliminate unnecessary manipulations, the induced $\mathrm{RSc} 0454$ protein was purified from the supernatant fraction (Fig. 2A), and the final concentration of purified $\mathrm{RSc} 0454$ was quantified as $0.25 \mu \mathrm{g} / \mu \mathrm{l}$. In LDH activity analyses, $4 \mu \mathrm{l}$ of purified protein showed an optimal reduced absorbance value of $A_{340}$ for every minute (Fig. 2B). LDH activity calculated from the first $3 \mathrm{~min}$ was about $20.7 \mathrm{U} / \mathrm{ml}$. In SDH activity analyses, $4 \mu \mathrm{l}$ of purified protein showed clear SDH activity. After incubation at $37^{\circ} \mathrm{C}$ for $30 \mathrm{~min}$ in the dark, purple blue precipitates were produced in the reaction mixture. Quantification analysis of SDH activity showed an $A_{548}$ value of 0.29 (Fig. 2C). Since the protein amount in $4 \mu \mathrm{l}$ purified sample was $1.0 \mu \mathrm{g}$, the specific activity of SDH was calculated as 0.29 . Therefore, the large FAD-linked oxidase RSc0454 showed both LDH and SDH activities.

\section{The RSc0454 gene is indispensable}

for pathogenicity by $R$. solanacearum.

To investigate the role of the $\mathrm{RSc} 0454$ gene in $R$. solanacearum pathogenicity, a deletion mutant was constructed via two steps of homolog recombination. The desired mutant was confirmed by PCR analysis (Supplementary Fig. S2). Inoculation of the mutant into tomato plant stems did not cause any wilt symptoms. At 3 days postinoculation (DPI), dark narrow streaks were visible on plant stems inoculated with wild-type GMI1000. At 5 DPI, the dark streaks expanded to petioles of closed leaves. At 18 DPI, all plants inoculated with wild-type GMI1000 had completely collapsed (Fig. 3A). The complemented strain restored the pathogenicity of the mutant to the wild type, and the disease index was similar to that of the wild type (Fig. 3B).

\section{The RSc0454 gene is involved} in cell dispersion in NB liquid medium.

Deletion of the RSc0454 gene did not slow growth in nutrient-rich broth (NB) medium. The bacterial growth speed of the mutant was not different than that of the wild type (Fig. 4A). When culturing strains to test pathogenicity, the mutant cells quickly aggregated at the bottom of the glass tube if the tube remained stationary. To determine whether this phenotype was caused by the deletion of the RSc0454 gene, the phenotypes were carefully studied. After $24 \mathrm{~h}$ of stationary incubation, the wild-type GMI1000 grew in a dispersed planktonic fashion in the liquid NB medium, and the cultures remained turbid. Since the RSc0454 deletion mutant cells were aggregated at the bottom of the glass tube, the liquid medium appeared transparent. The complemented strain restored the growing mode to that of the wild type (Fig. 4B). Considering that this altered growth mode was caused by the loss of $\mathrm{RSc} 0454$, pyruvate, lactate, succinate, and fumarate were added to the liquid medium. Application of these four carbon resources restored the dispersed growing mode but to varying degrees. Distinctive restoration was found in cultures supplemented with succinate or fumarate. Supplementation with pyruvate and lactate partially restored cell dispersion (Fig. 4B).

\section{A single DUF3683, LDH, or SDH domain cannot restore the altered pathogenicity of the mutant.}

Since supplementation of catalytic carbohydrates restored cell dispersion, we investigated whether a single $\mathrm{LDH}, \mathrm{SDH}$, or DUF3683 domain was able to restore the pathogenicity phenotype of the mutant. The predicted DUF3683, LDH, and SDH domains were expressed in pBBR1MCS-5 and used to generate CM-DUF, CM-LDH, and CM-SDH after introduction into the mutant (Fig. 5A). In comparison with the mutant strain complemented with full-length RSc0454, CM-DUF, CM-LDH, and CM-SDH did not cause wild-type symptoms on tomato plants. At 5 DPI, dark streaks expanded to the petioles of closed leaves on plants that were inoculated with full-length complemented or wild-type strains. CM-DUF, CM-LDH, and CM-SDH caused no symptoms at the inoculation site, similar to the mutant strain (Fig. 5B). Thus, the disease indexes of CM-DUF, CM-LDH, and CM$\mathrm{SDH}$ were similar to that of the mutant strain, remaining at zero over the 20-day observation period (Fig. 5C).
A

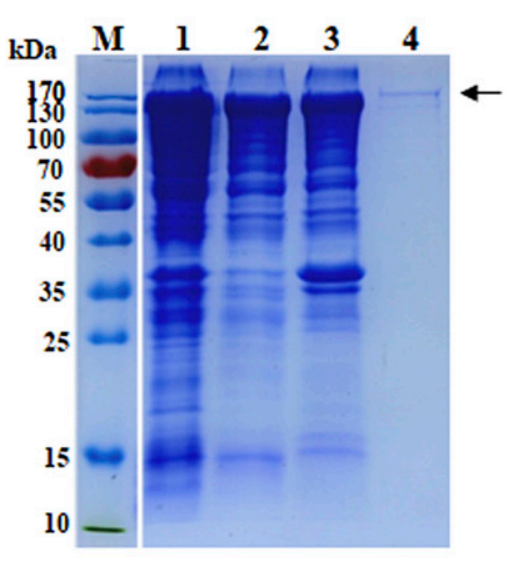

B

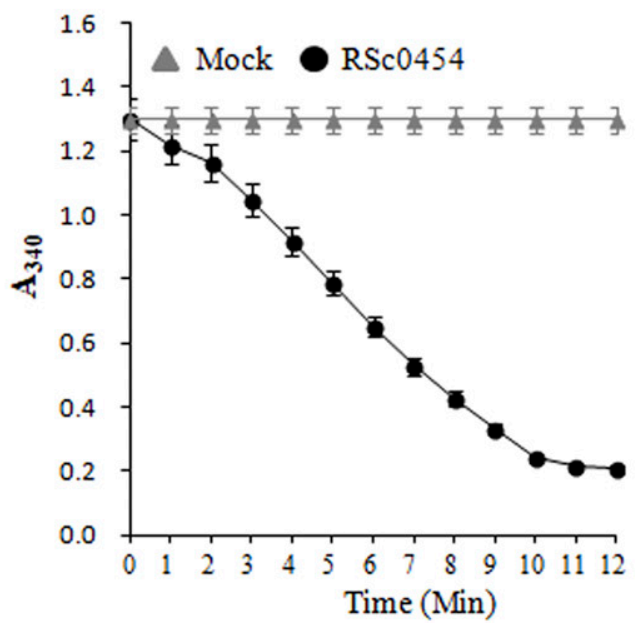

C

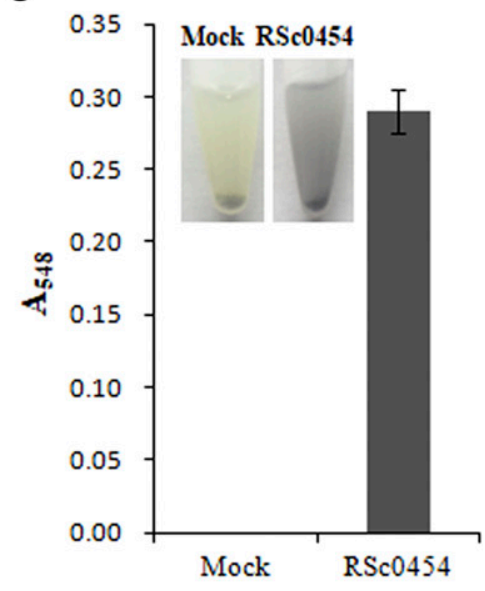

Fig. 2. Dehydrogenase activity assays of the RSc0454 protein. A, Sodium dodecyl sulfate-polyacrylamide gel electrophoresis of the RSc0454 protein purified from a prokaryotic expression system. The induced $\mathrm{RSc} 0454$ protein was purified on glutathione resin. A protein sample ( $2 \mu \mathrm{l})$ was applied for electrophoresis. Lane M, PageRuler prestained protein ladder; lane 1, total protein after sonication; lane 2, protein sample of supernatant after sonication; lane 3, protein sample of cell debris after sonication; lane 4, purified RSc0454 protein. B, Lactate dehydrogenase activity assay for the RSc0454 protein. The reaction was initiated by addition of $4 \mu \mathrm{l}$ of purified RSc0454 protein. All assays were performed in triplicate at room temperature. The decreased $A_{340}$ value was monitored every minute over the course of $12 \mathrm{~min}$. $\mathbf{C}$, Succinate dehydrogenase activity assay for the RSc0454 protein. The reaction was initiated by addition of $4 \mu \mathrm{l}$ of purified $\mathrm{RSc} 0454$ protein into the reaction buffer. Purple blue precipitates were scored after incubation for 30 min in the dark at $37^{\circ} \mathrm{C}$. Enzyme activity was quantitively measured at $A_{548}$ by absorption spectroscopy. In the mock control, the substrates sodium pyruvate and diethyl-succinate were omitted from the reaction system, respectively. 
The expression of the $\mathrm{RSc0454}$ gene is inducible.

To study the transcription pattern of the RSc0454 gene, its promoter was first predicted via neural network promoter prediction. A putative promoter sequence with a score of 0.81 was found from position -88 to position -138 upstream of the translation start codon of the RSc0454 gene. A 445-bp DNA fragment comprising the predicted promoter region was fused with the LacZ gene and finally cloned into the pHM1 to generate pHM-PLacZ. On NB agar plates supplied with $20 \mu \mathrm{g}$ of $\mathrm{X}$-gal per milliliter, RSc0454 promoter-derived LacZ activity in $R$. solanacearum was determined via the blue color of the colonies (Fig. 6A). The negative control, pHM-LacZ, showed no $L a c Z$ activity (Fig. 6A). LacZ activity reached 26.9 Miller units when $R$. solanacearum was cultured in NB medium. Supplementation with pyruvate and succinate did not affect promoter activity. In contrast, supplementation with lactate and fumarate induced promoter activities (Fig. 6B). Promoter activity was also induced in minimal medium M63, which showed an average of $30 \%$ enhancement relative to the activity in NB medium (Fig. 6B). mRNA levels were subsequently studied, and the data were consistent with the results of promoter-monitored $L a c Z$ activities. The relative expression levels increased in $R$. solanacearum cells cultured in NB medium that were supplemented with lactate and fumarate, as well
A
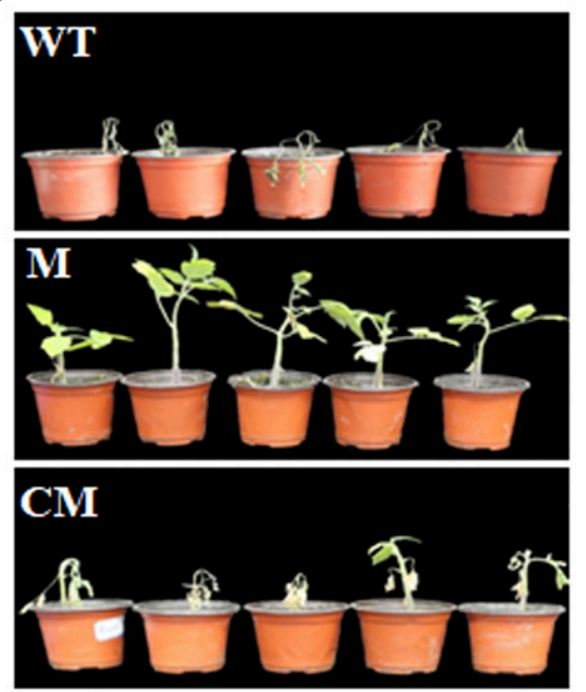

B

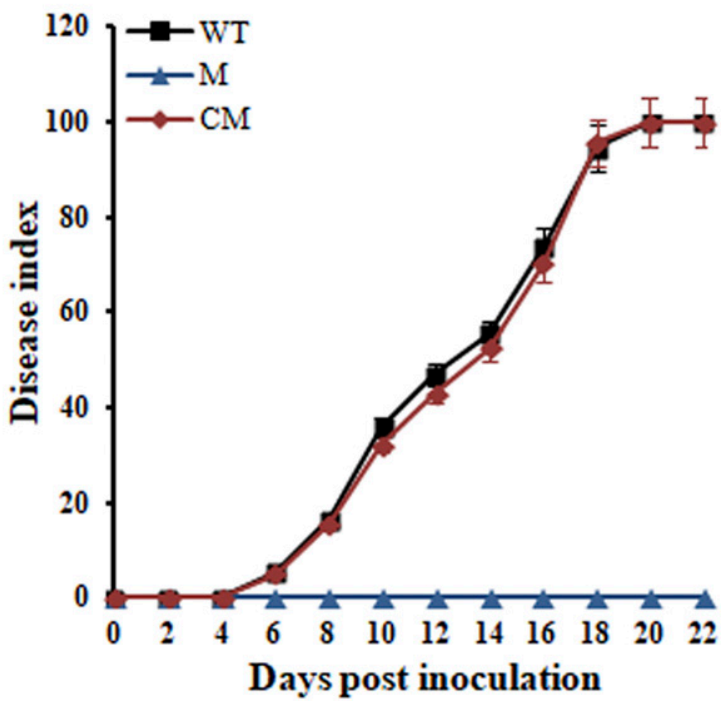

Fig. 3. Necessity of RSc0454 for Ralstonia solanacearum pathogenicity. A, Tomato plants were inoculated with the RSc0454 mutant. Cultured R. solanacearum was prepared to $\mathrm{OD}_{600}=0.3$ and inoculated onto tomato plants through stem cutting. The photos represent disease symptom development from one repeat at 15 days postinoculation. B, Disease severity of the RSc0454 mutant on tomato plants. Disease severity was rated every 2 days for 22 days, using a disease index scale. Data represent the means of four independent experiments, each experiment included five plants per treatment, and error bars represent standard errors. WT: wild-type GMI1000; M: RSc0454 mutant; CM: RSc0454 mutant complemented with pBB-RSc0454.

A

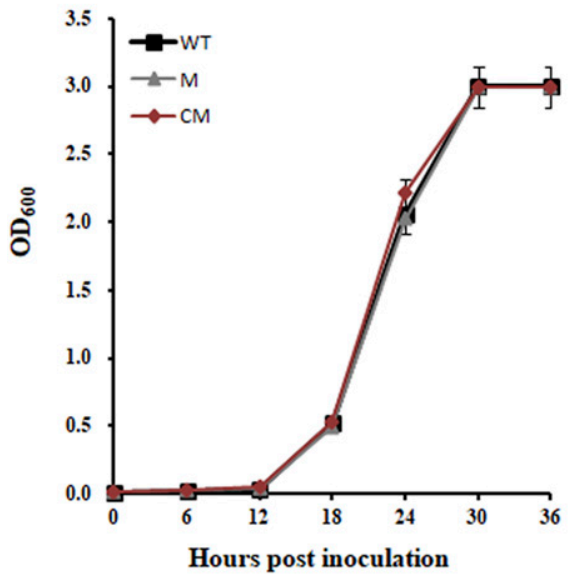

B

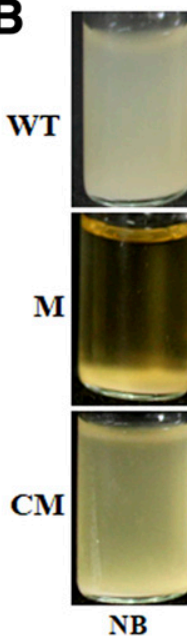

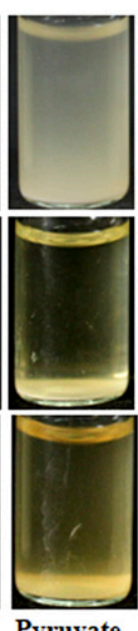

Pyruvate
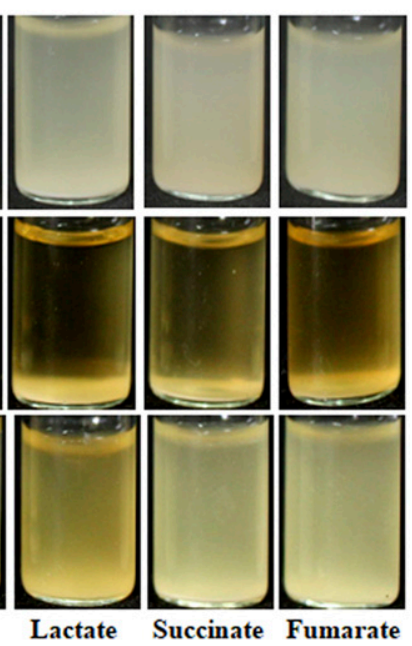

Fig. 4. Growth of the RSc0454 mutant in nutrient-rich broth (NB) medium. A, Growth curve of RSc0454 mutant in NB medium with shaking. Cultured cells were prepared to an $\mathrm{OD}_{600}=1.0$ in sterilized water and then subcultured $(1: 100)$ in fresh NB liquid medium. Growth rates were assessed by measuring $\mathrm{OD}_{600}$ values every $6 \mathrm{~h}$. All experiments were performed in triplicate and repeated three times. Error bars denote standard deviation of three experimental replicates. B, Growth mode of RSc0454 mutant in NB medium without shaking. Cultured cells were centrifuged at 6,000 rpm for 10 min and resuspended with fresh $\mathrm{NB}$ medium to an $\mathrm{OD}_{600}=1.5$. Cell suspensions were incubated in a stationary position at $28^{\circ} \mathrm{C}$ for $24 \mathrm{~h}$. To examine the roles of carbohydrates in cell aggregation, a final concentration of $40 \mathrm{mM}$ lactate, pyruvate, succinate, or fumarate was applied to the NB medium. All experiments were repeated three times WT: wild-type GMI1000; M: RSc0454 mutant; CM: RSc0454 mutant complemented with pBB-RSc0454. 
as in M63 medium (Fig. 6C). This indicated that the expression of $\mathrm{RSc} 0454$ was inducible in response to different $R$. solanacearum living conditions.

\section{Balanced transcription profile}

of $R S c 0454$, sdhBADC, and ldh genes in $R$. solanacearum.

Similar to other microbes, the standard SDH in $R$. solanacearum is composed of the following four subunits: SDHA, SDHB, SDHC, and SDHD. The four corresponding coding genes, $s d h A, s d h B, s d h C$, and $s d h D$, are clustered in the genome from position $2,170,085$ to position $2,173,377$. The D-lactate dehydrogenase gene $l d h$ is located in the genome from position 2,873,241 to position 2,874,659 (Fig. 7A). As LDH and SDH catalyze the dehydrogenation reactions under anaerobic growth conditions, the transcription profile of the RSc0454, $s d h B A D C$, and $l d h$ genes were studied in cells cultured in NB medium and in planta. Comparison of the results to the expression level in $\mathrm{NB}$ medium revealed the transcription of RSc0454 increased 1.2 -fold in $R$. solanacearum cells from tomato stems that showed wilt symptoms (Fig. 7B). The $s d h B A D C$ and $l d h$ genes showed higher transcription levels in cells cultured in NB medium, while they reduced their transcription level in planta (Fig. 7B).

\section{DISCUSSION}

This paper reports, for the first time, the role of the FAD-linked oxidase RSc0454 in the pathogenicity of plant pathogenic bacteria. R. solanacearum (previously named Pseudomonas solanacearum and Burkholderia solanacearum) is considered a species complex: a group of close, but genetically distinct, species (Genin and Denny 2012). Based on sequence similarity and phylogenetic analysis, homologous sequences of the RSc0454 gene characterized from Ralstonia species in this study are also present in the genomes of species of Pandoraea, Cupriavidus, Paraburkholderia, and Burkholderia. All species of these five genera possess the FAD-linked oxidase and can be isolated from soil environments (Anandham et al. 2010; Dobritsa and Samadpour 2016; Vandamme and Coenye 2004) For example, Paraburkholderia rhynchosiae has been isolated from root nodules of the plant Rhynchosia ferulifolia in South Africa (De Meyer et al. 2013). The homologies of the

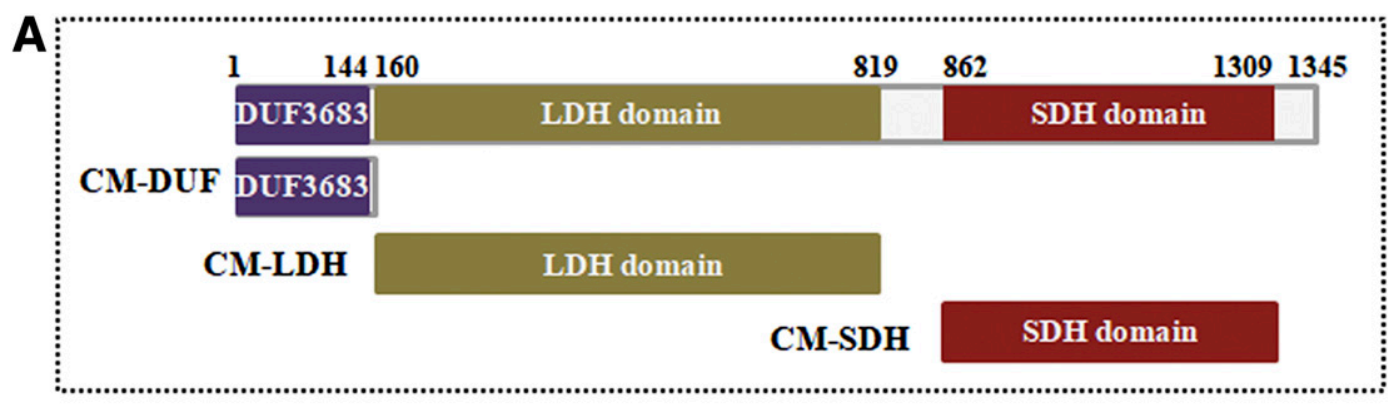

B
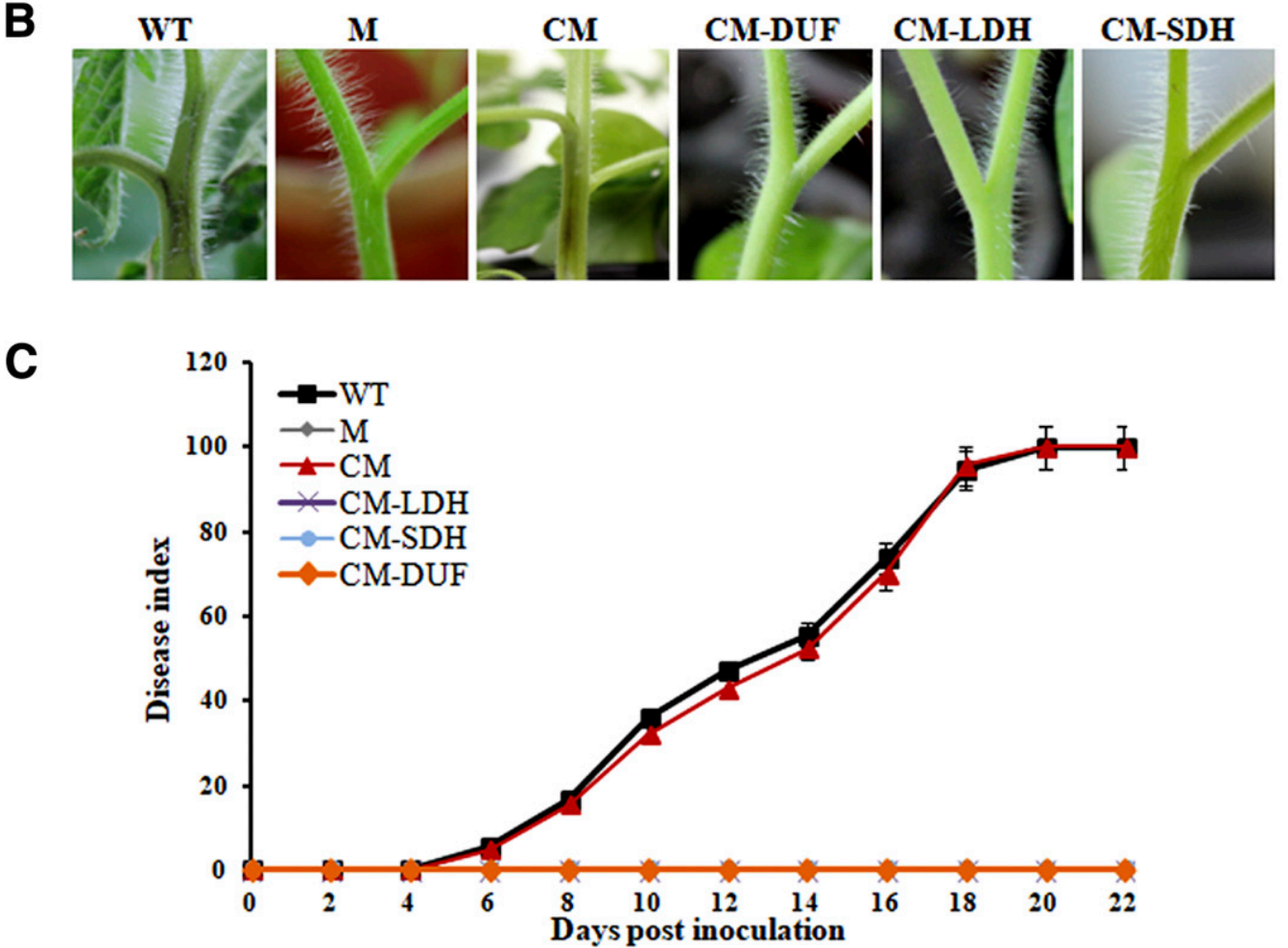

Fig. 5. Pathogenicity of RSc0454 mutant restored by DUF3683, lactate dehydrogenase (LDH), or succinate dehydrogenase (SDH) domain. A, Schematic diagram of the complementary construction expressing the predicted DUF3683, LDH, or SDH domain. The resulting recombinant constructs were introduced into the RSc0454 mutant to generate CM-DUF, CM-LDH, and CM-SDH, respectively. B, Disease symptoms on tomato plants caused by complemented strains at 5 days postinoculation. The cultured Ralstonia solanacearum was prepared to $\mathrm{OD}_{600}=0.3$ and inoculated onto tomato plants through stem cutting. C, Disease severity rated for 22 days after inoculation. Data represent the means of four independent experiments, each experiment included five plants per treatment, and error bars represent standard errors. WT: wild-type GMI1000; M: RSc0454 mutant; CM: RSc0454 mutant complemented with pBB-RSc0454. 
Cupriavidus genus are found in nine species. Except for C. alkaliphilus, $C$. nantongensis, and $C$. pinatubonensis, the remaining six species were reclassified from the genus Ralstonia (Vandamme and Coenye 2004). This particular distribution suggests that $\mathrm{RSc0454}$ plays a specific role during the life cycle of soilborne microbes, especially those with a close relationship to Ralstonia.

The FAD-linked oxidase RSc0454 in R. solanacearum is a large protein, which consists of three distinct domains. The $\mathrm{N}$-terminal DUF3683, LDH, and C-terminal SDH domains did not restore the pathogenicity of the RSc0454 mutant. This suggests that multiple functional domains of the RSc0454 protein are required for $R$. solanacearum to cause wilt disease in host plants. Therefore, RSc0454 may be involved in several biological processes in $R$. solanacearum. In addition to catalyzing the redox reaction directly, $\mathrm{LDH}$ and $\mathrm{SDH}$ are essential for virulence, cell proliferation, and membrane generation in microbes (Abdelbaset et al. 2017; Kim et al. 2017; Mercado-Lubo et al. 2008; Pecsi et al. 2014). The respiratory proteins are localized to both the integral and peripheral membranes to execute their function. $\mathrm{LDH}$ is a peripheral membrane protein, while SDH is an integral membrane subunit (Futai 1973). A peripheral membrane protein and an integral membrane protein differ not only in location and function, but also in the nature of their interaction with the lipid bilayer (Cymer et al. 2015). It would be intriguing to experimentally determine the subcellular location of RSc0454, which would provide a further clue to understanding its biological function.

Aside from a large number of virulence factors, $R$. solanacearum must acquire nutrients from host plants for cell proliferation. $R$. solanacearum is able to utilize available nutrients, especially carbohydrates, from xylem sap (Hayward 1991; Zuluaga et al.
2013). Compared with culturing conditions, a significant proportion of genes is induced after the bacterium enters the host xylem, including those involved in both sucrose uptake and catabolism (Jacobs et al. 2012). This guarantees that $R$. solanacearum can multiply very quickly, thus reaching high cell densities, which is required for the pathogen to cope with the host immune system. During replication in the xylem, a quorum-sensing dependent regulatory protein, $\mathrm{PhcA}$, is responsible for controlling the trade-off between bacterial proliferation and virulence factor production (Peyraud et al. 2016). Furthermore, chemotaxis enables $R$. solanacearum to move toward preferable nutritional compounds that are abundant in planta, such as D-glucose (Peyraud et al. 2016). These data show that carbohydrate assimilation and metabolism are essential for $R$. solanacearum multiplication in planta. In this report, the large oxidase RSc0454 was found to be indispensable for $R$. solanacearum pathogenicity. Considering that $\mathrm{RSc} 0454$ is involved in the dehydrogenation reaction, we propose that specific redox reactions are critical for Ralstonia to establish successful infection.

Quorum sensing enables bacteria to act in a coordinated manner by perceiving low-molecular-weight signal molecules (von Bodman et al. 2003). This facilitates survival of the individual bacterium in nature and aids in the colonization of the host through cooperative group behavior (Parsek et al. 1999). In addition to acyl homoserine lactone molecules (Kumar et al. 2016), the unusual quorum-sensing signals 3-hydroxy palmitic acid methyl ester and methyl 3-hydroxymyristate are synthesized by $R$. solanacearum to control cell density and relative virulence factors (Flavier et al. 1997; Kai et al. 2015). In the plant pathogenic bacterium Xanthomonas campestris pv. campestris, several $r p f$ genes are involved in the biosynthesis and signal

A

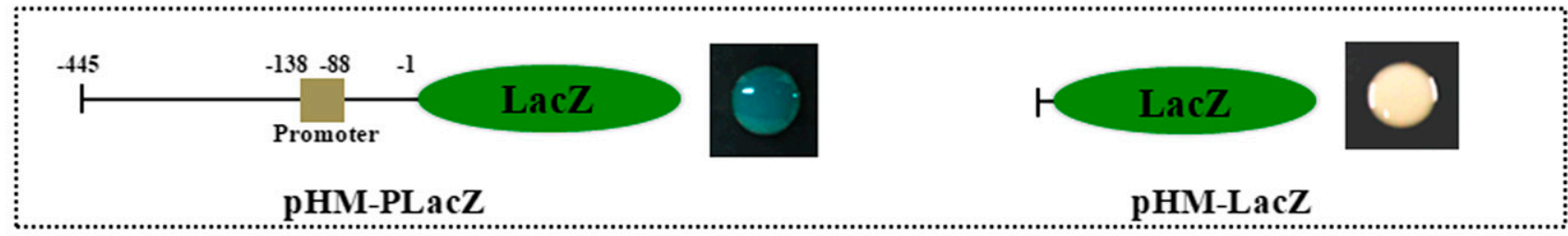

B

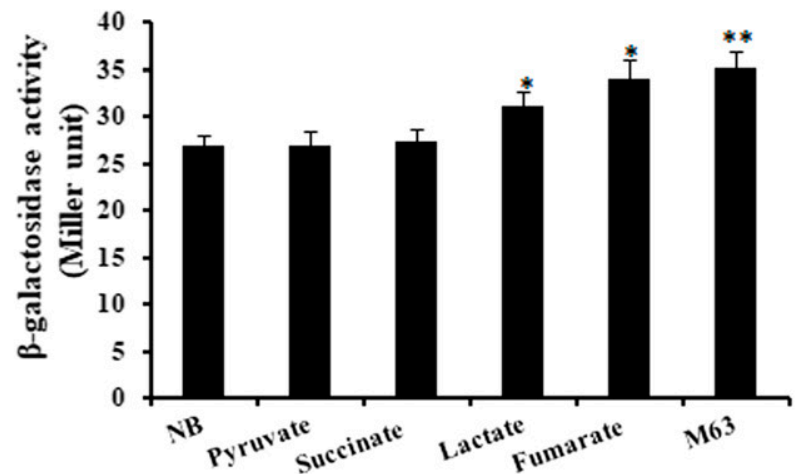

C

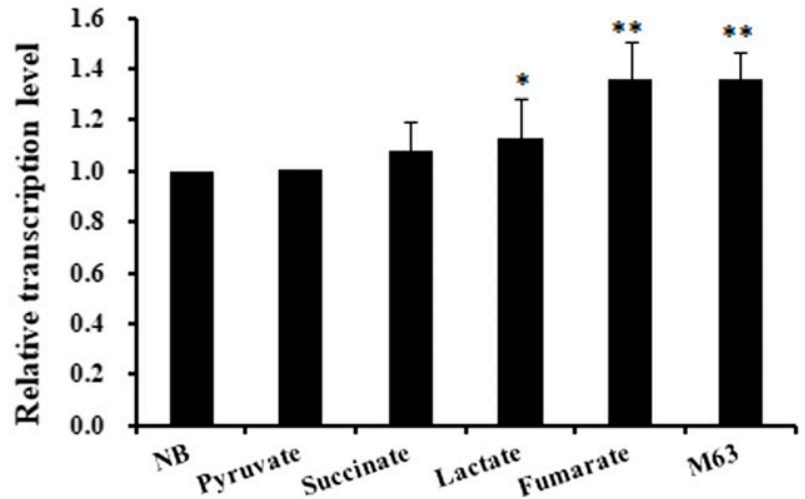

Fig. 6. Transcription of RSc0454 in response to different living conditions. A, Cloning of the RSc0454 promoter fused with the LacZ gene. A 445 -bp promoter region was fused with the LacZ gene to generate pHM-PLacZ. After transforming into Ralstonia solanacearum GMI1000, promoter activity was assayed on nutrient agar (NA) plates supplemented with $20 \mu \mathrm{g}$ of X-gal per milliliter. The construct pHM-LacZ (without promoter ahead of the LacZ gene) was used as the negative control. B, Quantification of promoter-monitored $\beta$-galactosidase activities in $R$. solanacearum. Quantitative analysis of $\beta$-galactosidase activity was performed according to the standard Miller method. $R$. solanacearum carrying pHM-PLacZ was cultured in both nutrient-rich broth (NB) and minimal medium M63. To study the effects of carbohydrates on the transcription of RSc0454, $40 \mathrm{mM}$ lactate, pyruvate, succinate, or fumarate was added to the NB medium. C, qRT-PCR analysis of RSc0454 transcription levels. Total RNA was extracted from wild-type R. solanacearum GMI1000 cultured in both NB and minimal medium M63. The expression of gyrA was used as the internal control. To compare the induction changes, the expression level of RSc0454 in NB liquid medium was set as " 1 ." Error bars indicate standard deviations, and asterisks $(*)$ denote statistical significance $(*, P<0.05 ; * *, P<0.01)$. 
transduction of small quorum-sensing signal molecules (Barber et al. 1997). Compared with wild-type growth that follows a dispersed planktonic fashion, the $r p f F, r p f G$, and $r p f C$ mutants grow as matrix-enclosed aggregates. Addition of quorumsensing signal molecules triggers dispersion of the aggregates formed by the $r p f F$ mutant strain (Dow et al. 2003). The growth phenotype of the RSc0454 mutant showed a growth mode similar to $X$. campestris pv. campestris rpf mutants. When cultured in NB medium without shaking, cells aggregated at the bottom of the test tube. Thus, whether deletion of RSc0454 affects quorum-sensing signal production requires further clarification.

Both transcription and translation of $\mathrm{LDH}$ and SDH in microbes vary in response to various environmental conditions. The transcription of $l d h A$ in Escherichia coli is induced approximately 10 -fold in anaerobically grown cultures at acidic pH (Mat-Jan et al. 1989). LDH can also be regulated by the relative concentrations of its substrates (Summermatter et al. 2013). In medicine, LDH often shows false-positive results for high levels in blood samples due to erythrocyte damage and, thus, is used as a marker for tissue breakdown (Kato et al.
2006). Plant SDH is allosterically regulated by the binding of oxaloacetate and is activated by ATP. SDH levels are increased by aerobic conditions and decreased by anaerobiosis in the presence of glucose (Huang and Millar 2013). In E. coli, an sdhLacZ fusion experiment has revealed that the SDH level varies from the type of carbon substrate utilized (Park et al. 1995). The data in this report clearly show that the transcription of $\mathrm{RSc} 0454$ was induced by addition of carbohydrates, as well as by culture, under nutrient-limited conditions. The enhanced expression of the $\mathrm{RSc0454}$ gene in response to diverse living environments was similar to expression of $s d h$ and $l d h$ genes.

The soilborne bacterium $R$. solanacearum gains access to the host root system through natural wounds caused by the emergence of lateral roots or through wounds incurred as roots grow through soil. During the invasion stage, flagella-driven swimming is important for both ecological fitness and host colonization (Yao and Allen 2006, 2007). After entering the root, bacteria move to the root vascular system, where they multiply in the xylem and subsequently spread into shoots (Hayward $1991)$. In the plant xylem, $R$. solanacearum cells live under low $\mathrm{O}_{2}$ content and limited nutrient availability conditions in the
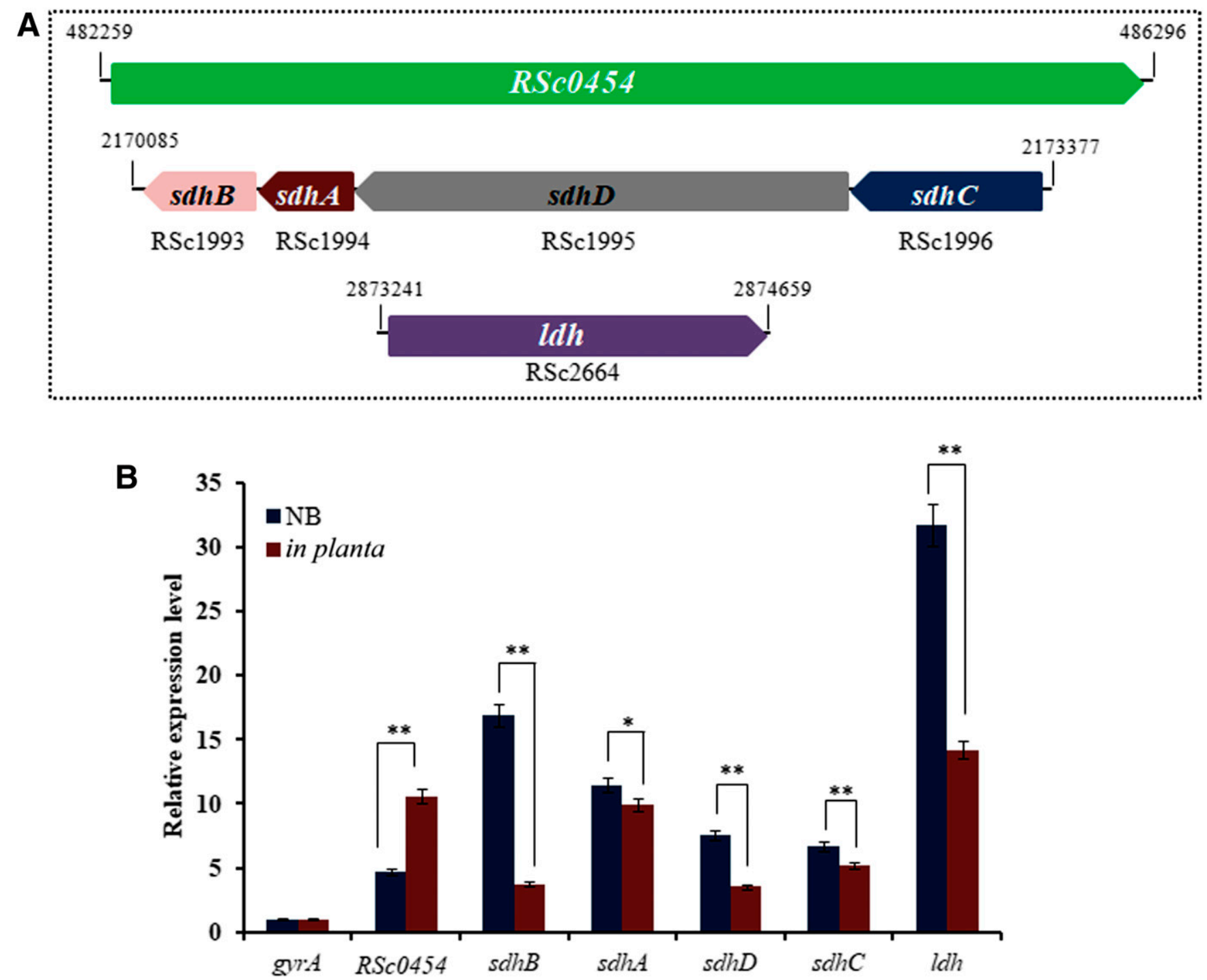

Fig. 7. qRT-PCR analysis of RSc0454, sdhBADC, and $l d h$ in Ralstonia solanacearum. A, Locations of RSc0454, sdhBADC, and $l d h$ genes in the genome of $R$. solanacearum GMI1000. B, qRT-PCR analysis of transcription levels of RSc0454, sdhBADC, and ldh genes in R. solanacearum GMI1000 cultured in NB medium and in planta. The transcription level of each gene was studied by comparing cells cultured in NB medium to cells cultured in tomato stems. The expression of $g y r A$ was used as an internal control and was set as "1" in either RNA sample. Error bars indicate standard deviations, and asterisks (*) denote statistical significance $(*, P<0.05 ; * *, P<0.01)$. 
xylem fluid. With the exception of large numbers of genes involved in carbon and nitrogen resource metabolism, transcriptions of carbohydrate dehydrogenases vary (Jacobs et al. 2012). qRT-PCR analysis here showed that both the $s d h B A D C$ and $l d h$ genes had decreased transcription levels when the bacterium colonized inside the xylem compared with culture in liquid medium. In contrast, the transcription of RSc0454 was enhanced in planta. This particular transcription pattern suggested that a complex redox reaction balance had occurred during $R$. solanacearum infection. During the invasion stage, $R$. solanacearum cells live under comparatively high $\mathrm{O}_{2}$ content conditions. $R$. solanacearum mainly relies on $\mathrm{SDH}$ and LDH to dehydrogenize lactate and succinate, and when entering the xylem, RSc0454 plays a dominant role in the dehydrogenation of both lactate and succinate (Fig. 8).

Due to the necessity of RSc0454 for pathogenicity, we presumed RSc0454 would be an ideal target to explore novel bactericides for the control of bacterial wilt pathogens. Several chemicals show promising inhibition effects on LDH activities. For instance, the LDH inhibitor galloflavin has been reported to suppress liver damage and improve the survival of mice with acute liver failure (Ferriero et al. 2018). SDH is an essential component of the cellular respiratory chain and tricarboxylic acid cycle and is also the target for fungicides. By using SDH as a target, a novel pyrazol-benzoic scaffold inhibitor was identified that displayed excellent activity against Rhizoctonia solani, Sclerotinia sclerotiorum, and Pyricularia grisea (Yao et al. 2017). A number of SDH inhibitors have been developed as agricultural fungicides and display broad-spectrum activity against fungal species (Sierotzki and Scalliet 2013). RSc0454

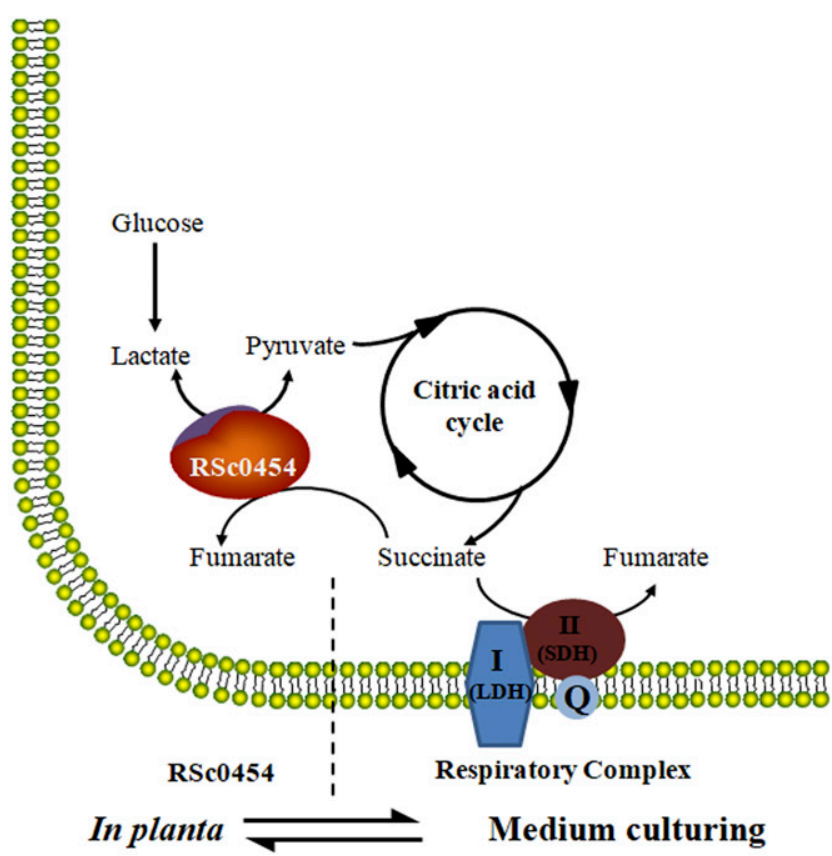

Fig. 8. A balanced transcription profile of the genes that contribute to lactate dehydrogenase (LDH) and succinate dehydrogenase (SDH) activities in Ralstonia solanacearum GMI1000. The $l d h$ and $s d h B A D C$ genes code for corresponding respiratory complexes I and II, respectively. When culturing in liquid medium, the transcriptions of $l d h$ and $s d h B A D C$ are at a high level, while the transcription of RSc0454 remains at a low level. Inside the host xylem, the transcriptions of $l d h$ and $s d h B A D C$ are distinctly decreased. In contrast, the transcription of RSc0454 increases to a higher level. This particular transcription profile suggests that $R$. solanacearum GMI1000 relies mainly on RSc0454 to catalyze LDH and SDH when living in the host xylem. is distributed in a limited number of microbe species, and the inhibitors identified should exhibit high specificity.

In conclusion, this study demonstrated that the oxidase encoded by $\mathrm{RSc} 0454$ is essential for the wild-type pathogen $R$. solanacearum to cause disease on a tomato host. This is the first study to show that this oxidase shows alternative LDH and SDH activities and that it is transcribed in coordination with $s d h B A D C$ and $l d h$ genes. The pathogenic necessity of this oxidase indicates a potential target for the identification of small chemical inhibitors for the development of novel bactericides.

\section{MATERIALS AND METHODS}

\section{Bacterial strains and plasmids.}

All plasmids and strains used in this study are listed in Supplementary Table S1. E. coli strains were grown at $37^{\circ} \mathrm{C}$ in Luria-Bertani medium. $R$. solanacearum strains were grown at $28^{\circ} \mathrm{C}$ in NB medium. To study the expression of the RSc0454 gene under nutrient-limited conditions, $R$. solanacearum also was cultured in the minimal medium M63. The following types and concentrations of antibiotics were used: kanamycin $(\mathrm{Km})$, $50 \mu \mathrm{g} / \mathrm{ml}$; spectinomycin (Sp), $20 \mu \mathrm{g} / \mathrm{ml}$; gentamycin $(\mathrm{Gm})$, $10 \mu \mathrm{g} / \mathrm{ml}$; and ampicillin (Ap), $50 \mu \mathrm{g} / \mathrm{ml}$.

\section{SDH and LDH activity assays.}

The full length of RSc0454 contains a SphI site from position 1,264 to position 1,270 . To clone the full length of $\mathrm{RSc} 0454$ for prokaryotic expression, the primer set E1.F/E1.R was used in PCR to amplify a 1,280-bp DNA fragment of RSc0454 from the translation start codon to the SphI site. The BamHI and HindIII cutting sites were introduced into the $5^{\prime}$ termini of primers E1.F and E1.R, respectively (Supplementary Table S2). The reverse primer E1.R was designed to cover the SphI site. The 1,280-bp PCR product was inserted into the pMD19-T simple vector (TaKaRa), thus generating pMD19E1. A 2,778-bp DNA fragment of RSc0454 from the SphI site to the stop codon was then amplified using the primer set E2.F/E2.R. A HindIII cutting site was introduced into the E2.R primer at the $5^{\prime}$ terminus. The PCR product was inserted into pMD19-C1 at SphI-HindIII sites to generate pMD19-E1E2, within which the full-length RSc0454 gene was successfully cloned into the pMD19-T simple vector. The entire coding sequence of RSc0454 was digested from pMD19-E1E2 and was subsequently inserted into pET41a $(+)$ at BamHI and HindIII sites. The resulting construct was transformed into BL21(DE3) cells for induction with $1.0 \mathrm{mM}$ isopropyl- $\beta$-D-thiogalactopyranoside. Bacterial cells were harvested and resuspended in $10 \mathrm{mM}$ phosphatebuffered saline. After sonication, insoluble cell debris was separated from the supernatant by centrifugation. RSc0454 proteins were purified from the supernatant on a glutathione resin (Genescript). Protein samples were analyzed using $10 \%$ sodium dodecyl sulfate-polyacrylamide gel electrophoresis, and the protein concentration was determined using the Bradford method.

The LDH assay was performed as previously described (Karamanos 2014). The reaction was initiated by addition of $4 \mu \mathrm{l}$ of purified $\mathrm{RSc} 0454$ protein to the assay mixtures (1.0 mM sodium pyruvate, $0.2 \mathrm{mM}$ NADH, $50 \mathrm{mM}$ Tris-buffer, $\mathrm{pH}$ 7.5). The decreased $A_{340}$ value was monitored every minute over $12 \mathrm{~min}$. All assays were performed in triplicate at room temperature. The LDH activity per milliliter was obtained using the formula: $\left(\Delta A_{340}\right) /\left(4 \mu \mathrm{l} \times 10^{-3}\right)$. SDH activity was assayed using the nitroblue tetrazolium (NBT) method (Ezawa and Ogata 1979). NBT is soluble in water, where it shows a pale yellow color. When using NBT as a hydrogen acceptor to 
receive the hydrogen from sodium succinate, purple-blue precipitates form in the reaction mixture $(12.3 \mathrm{mM}$ diethyl-succinate, $1.2 \mathrm{mM}$ NBT, $0.2 \mathrm{mM}$ 1-methoxy5-methylphenazine methyl sulfate, $50 \mathrm{mM}$ Tris- $\mathrm{HCl}, \mathrm{pH}$ 7.6). Followed by addition of $4 \mu \mathrm{l}$ of purified enzyme, the reaction mixtures were incubated at $37^{\circ} \mathrm{C}$ for $30 \mathrm{~min}$ in the dark. The enzyme activity was determined by the formation of nitro blue diformazan due to NBT reduction and was measured at $A_{548}$. One unit of activity was defined as a reduction of one $A_{548}$ value within $30 \mathrm{~min}$ : Specific activity = Activity unit $/ \mathrm{mg}$ protein.

\section{Mutant generation.}

The deletion mutant was generated using the suicide vector pK18mobsacB according to a previously described method (Zhang et al. 2015). The primer pairs M1.F/M1.R and M2. F/M2.R were used to amplify two flanking DNA fragments of the RSc0454 gene. The flanking sequences were fused by overlapping PCR and then inserted into the suicide vector pK18mobsacB at $\mathrm{XbaI}-\mathrm{SphI}$ sites to generate pK18-RSc0454. After the recombinant construct was electrotransformed into wild-type $R$. solanacearum GMI1000, a deletion mutant was produced by two steps of homolog recombination (Zhang et al. 2015). The primer set M1.F/M2.R was used to amplify a shortened fragment of the mutant genome DNA.

\section{Construction of complementary plasmids.}

Similar to the construction strategy for prokaryotic expression, the full-length RSc0454 was cloned into pBBR1MCS-5 to generate the complementation construct pBB-RSc0454. The primer set C1.F/C1.R was used in PCR to amplify the 1,573-bp DNA fragment containing a 295-bp promoter region and a 1,278-bp RSc0454 partial sequence from the translation start site. The primer C2.R was combined with the primer E2.F to amplify the 2,778-bp DNA fragment of RSc0454 from the SphI site to the stop codon. After both DNA fragments were fused in pMD19-T simple, the RSc0454 coding sequence and its promoter were removed in pBBR1MCS-5 at the HindIII and BamHI sites to generate the complementation construct pBB-RSc0454. In this complementation analysis construct, the Rsc0454 gene was expressed under the control of its native promoter.

A 943-bp DNA fragment containing promoter region and $\mathrm{N}$ terminal DUF3683 was amplified using PCR and primer set DUF.F/DUF.R. The stop codon was introduced into the reverse primer DUF.R. The PCR product was cloned in pBBR1MCS-5 at KpnI-BamHI sites, generating pBB-DUF. To generate single LDH or SDH domain complementation constructs, a 445-bp DNA fragment containing a promoter region upstream of the RSc0454 translation start codon was first cloned in pBBR1MCS-5 at KpnI-XhoI sites. Then, the sequence encoding the predicted LDH or SDH domain was cloned in HindIII$B a m \mathrm{HI}$ and BamHI-SacI sites, respectively. For each primer set that amplifies the LDH or SDH domain, the translation start and stop codons were introduced into forward and reverse primers. The resulting $\mathrm{pBB}-\mathrm{DUF}, \mathrm{pBB}-\mathrm{LDH}$, and $\mathrm{pBB}-\mathrm{SDH}$ were electrotransformed into the $\mathrm{RSc} 0454$ mutant to produce CM-DUF, CM-LDH, and CM-SDH.

\section{Pathogenicity on the tomato plant.}

Pathogenicity assays were conducted on susceptible tomato plants (Lycopersicon esculentum Mill. 'Hongyangli') using the stem inoculation method (Yao and Allen 2006). The cultured $R$. solanacearum cells $\left(\mathrm{OD}_{600}=0.3\right)$ were inoculated into the stem with a cut. Wilt symptoms were scored every 2 days postinoculation over 3 weeks. Disease severity and the percent severity index were recorded using a previously described method (Sun et al. 2017). This experiment was repeated at least four times, and five plants were used for every strain per replicate.

\section{Measurement of bacterial growth.}

The Ralstonia strains were cultured in NB medium on a shaker until they reached an $\mathrm{OD}_{600}=1.0$. Next, cells were subcultured (1:100) in $4 \mathrm{ml}$ of fresh $\mathrm{NB}$ and incubated for another $16 \mathrm{~h}$ until they reached an $\mathrm{OD}_{600}=0.6$. After centrifuging at $6,000 \mathrm{rpm}$ for $10 \mathrm{~min}$ at $4{ }^{\circ} \mathrm{C}$, the obtained cell pellets were resuspended with sterilized water to an $\mathrm{OD}_{600}=$ 1.0. The cell suspension was subcultured $(1: 100)$ in NB liquid medium, and bacterial growth was measured by determining the $\mathrm{OD}_{600}$ value every $6 \mathrm{~h}$. All experiments were repeated three times.

\section{Cell aggregation investigation.}

The Ralstonia strains were cultured in NB medium to an $\mathrm{OD}_{600}=1.0$. Next, cells were centrifuged at $6,000 \mathrm{rpm}$ for 10 min at $4{ }^{\circ} \mathrm{C}$ and resuspended with fresh $\mathrm{NB}$ medium to an $\mathrm{OD}_{600}=1.5$. Cells $(2 \mu \mathrm{l})$ were moved into $20-\mathrm{ml}$ glass tubes and kept stationary at $28^{\circ} \mathrm{C}$ for $24 \mathrm{~h}$. To examine the roles of carbohydrates in cell aggregation, a final concentration of $40 \mathrm{mM}$ lactate, pyruvate, succinate, or fumarate was added to the NB medium. All experiments were repeated three times.

\section{RNA extraction and qRT-PCR.}

The RNAprep Pure Cell/Bacteria kit (Tiangen Biotech) was used to extract total RNA from $R$. solanacearum cultured in NB medium. To evaluate the gene expression in planta, total RNAs from $R$. solanacearum were extracted from diseased tomato plant stems using the Plant RNA kit (Omega). Contaminated DNA was removed by digestion with RNAase-free DNAase I (TaKaRa). All RNA samples were quantified by measuring their $\mathrm{OD}_{260} / \mathrm{OD}_{280}$ ratios and then analyzed by gel electrophoresis for detection of intact RNA. The first-strand cDNA was synthesized with the GoScript reverse-transcription kit (Promega). PCR was conducted on the CFX Connect RealTime PCR Detection system (Bio-Rad) using the iTaq Universal SYBR Green Supermix (Bio-Rad). The following PCR thermal cycle conditions were used: denaturation at $95^{\circ} \mathrm{C}$ for 30 $\mathrm{s}$, and 40 cycles of $95^{\circ} \mathrm{C}$ for $5 \mathrm{~s}$ and $58^{\circ} \mathrm{C}, 20 \mathrm{~s}$. The expression of $g y r A$ was used as an internal control. Relative expression level and statistical analysis were conducted using CFX Maestro software (Bio-Rad). The average threshold cycle was normalized to the internal control in Mode of Normalized expression $(\Delta \Delta \mathrm{Cq})$.

\section{Promoter-monitored $\beta$-galactosidase activity.}

The 445-bp promoter region of the RSc0454 gene was fused with the LacZ gene in the pLacZ-Basic vector (Clontech) at KpnI-XhoI sites. Next, the promoter region, in combination with the LacZ gene, was removed into the cosmid pHM1 by digestion with KpnI-SalI. The resulting pHM-PLacZ was introduced into wild-type $R$. solanacearum GMI1000 for promoter-monitored $\mathrm{LacZ}$ activity analysis. The 4.6-kb KpnISalI fragment containing the $L a c Z$ gene was digested from the pLacZ-Basic empty vector (Clontech) and cloned into pHM1 as a negative control. The control construct pHM-LacZ does not contain a promoter in front of the LacZ gene. The wild-type $R$. solanacearum GMI1000 strain carrying either construct was tested for promoter activity. The cultured cells were prepared to an $\mathrm{OD}_{600}=1.5$. Cells $(2 \mu \mathrm{l})$ were spotted on NB agar plates supplied with $20 \mu \mathrm{g}$ of $\mathrm{X}$-gal per milliliter, and $\beta$-galactosidase activity was determined via colony color at 2 days postinoculation. The quantitative analysis of $\beta$-galactosidase activity was performed according to the standard procedures described by Miller (Miller 1972). 


\section{Sequence analysis.}

BLASTP analysis in GenBank retrieved homologous sequences from related microbes using the $\mathrm{RSc} 0454$ protein sequence. Based on the amino acid sequence, phylogenetic analyses were performed using the neighbor-joining method implemented in MEGA5 (Tamura et al. 2011). The conserved domains were obtained following a search against the Conserved Domain Database deposited in GenBank. Threedimensional structure modeling was conducted with RaptorX. The promoter sequence was predicted using the Berkeley Drosophila Genome Project promoter prediction tool.

\section{LITERATURE CITED}

Abdelbaset, A. E., Fox, B. A., Karram, M. H., Abd Ellah, M. R., Bzik, D. J., and Igarashi, M. 2017. Lactate dehydrogenase in Toxoplasma gondii controls virulence, bradyzoite differentiation, and chronic infection. PLoS One 12:e173745.

Acin-Perez, R., and Enriquez, J. A. 2014. The function of the respiratory supercomplexes: The plasticity model. Biochim. Biophys. Acta 1837: 444-450.

Alric, J., and Johnson, X. 2017. Alternative electron transport pathways in photosynthesis: A confluence of regulation. Curr. Opin. Plant Biol. 37: $78-86$.

Anandham, R., Indiragandhi, P., Kwon, S. W., Sa, T. M., Jeon, C. O., Kim, Y. K., and Jee, H. J. 2010. Pandoraea thiooxydans sp. nov., a facultatively chemolithotrophic, thiosulfate-oxidizing bacterium isolated from rhizosphere soils of sesame (Sesamum indicum L.). Int. J. Syst. Evol. Microbiol. 60:21-26.

Barber, C. E., Tang, J. L., Feng, J. X., Pan, M. Q., Wilson, T. J., Slater, H., Dow, J. M., Williams, P., and Daniels, M. J. 1997. A novel regulatory system required for pathogenicity of Xanthomonas campestris is mediated by a small diffusible signal molecule. Mol. Microbiol. 24:555-566.

Bunch, P. K., Mat-Jan, F., Lee, N., and Clark, D. P. 1997. The $l d h A$ gene encoding the fermentative lactate dehydrogenase of Escherichia coli. Microbiology 143:187-195.

Cymer, F., von Heijne, G., and White, S. H. 2015. Mechanisms of integral membrane protein insertion and folding. J. Mol. Biol. 427:999-1022.

Dalsing, B. L., Truchon, A. N., Gonzalez-Orta, E. T., Milling, A. S., and Allen, C. 2015. Ralstonia solanacearum uses inorganic nitrogen metabolism for virulence, ATP production, and detoxification in the oxygen-limited host xylem environment. MBio 6:e02471-14.

De Meyer, S. E., Cnockaert, M., Ardley, J. K., Trengove, R. D., Garau, G., Howieson, J. G., and Vandamme, P. 2013. Burkholderia rhynchosiae sp. nov., isolated from Rhynchosia ferulifolia root nodules. Int. J. Syst. Evol. Microbiol. 63:3944-3949.

Dobritsa, A. P., and Samadpour, M. 2016. Transfer of eleven species of the genus Burkholderia to the genus Paraburkholderia and proposal of Caballeronia gen. nov. to accommodate twelve species of the genera Burkholderia and Paraburkholderia. Int. J. Syst. Evol. Microbiol. 66: 2836-2846.

Dow, J. M., Crossman, L., Findlay, K., He, Y. Q., Feng, J. X., and Tang, J. L. 2003. Biofilm dispersal in Xanthomonas campestris is controlled by cell-cell signaling and is required for full virulence to plants. Proc. Natl. Acad. Sci. U.S.A. 100:10995-11000.

Ezawa, I., and Ogata, E. 1979. $\mathrm{Ca}^{2+}$-induced activation of succinate dehydrogenase and the regulation of mitochondrial oxidative reactions. J. Biochem. 85:65-74.

Fatima, U., and Senthil-Kumar, M. 2015. Plant and pathogen nutrient acquisition strategies. Front. Plant Sci. 6:750.

Ferriero, R., Nusco, E., De Cegli, R., Carissimo, A., Manco, G., and BrunettiPierri, N. 2018. Pyruvate dehydrogenase complex and lactate dehydrogenase are targets for therapy of acute liver failure. J. Hepatol. 69:325-335.

Flavier, A. B., Clough, S. J., Schell, M. A., and Denny, T. P. 1997. Identification of 3-hydroxypalmitic acid methyl ester as a novel autoregulator controlling virulence in Ralstonia solanacearum. Mol. Microbiol. 26:251-259.

Flores-Cruz, Z., and Allen, C. 2009. Ralstonia solanacearum encounters an oxidative environment during tomato infection. Mol. Plant-Microbe Interact 22:773-782.

Futai, M. 1973. Membrane D-lactate dehydrogenase from Escherichia coli. Purification and properties. Biochemistry 12:2468-2474.

Gao, C., Wang, Y., Zhang, Y., Lv, M., Dou, P., Xu, P., and Ma, C. 2015. NAD-independent L-lactate dehydrogenase required for L-lactate utilization in Pseudomonas stutzeri A1501. J. Bacteriol. 197:2239-2247.
Garvie, E. I. 1980. Bacterial lactate dehydrogenases. Microbiol. Rev. 44: 106-139.

Genin, S., and Denny, T. P. 2012. Pathogenomics of the Ralstonia solanacearum species complex. Annu. Rev. Phytopathol. 50:67-89.

Grimm, S. 2013. Respiratory chain complex II as general sensor for apoptosis. Biochim. Biophys. Acta 1827:565-572.

Hayward, A. C. 1991. Biology and epidemiology of bacterial wilt caused by Pseudomonas solanacearum. Annu. Rev. Phytopathol. 29:65-87.

Heikal, A., Nakatani, Y., Dunn, E., Weimar, M. R., Day, C. L., Baker, E. N., Lott, J. S., Sazanov, L. A., and Cook, G. M. 2014. Structure of the bacterial type II NADH dehydrogenase: A monotopic membrane protein with an essential role in energy generation. Mol. Microbiol. 91:950-964.

Huang, S., and Millar, A. H. 2013. Succinate dehydrogenase: the complex roles of a simple enzyme. Curr. Opin. Plant Biol. 16:344-349.

Jacobs, J. M., Babujee, L., Meng, F., Milling, A., and Allen, C. 2012. The in planta transcriptome of Ralstonia solanacearum: Conserved physiological and virulence strategies during bacterial wilt of tomato. MBio 3: e00114-12.

Janssen, S., Schäfer, G., Anemüller, S., and Moll, R. 1997. A succinate dehydrogenase with novel structure and properties from the hyperthermophilic archaeon Sulfolobus acidocaldarius: Genetic and biophysical characterization. J. Bacteriol. 179:5560-5569.

Kai, K., Ohnishi, H., Shimatani, M., Ishikawa, S., Mori, Y., Kiba, A., Ohnishi, K., Tabuchi, M., and Hikichi, Y. 2015. Methyl 3-hydroxymyristate, a diffusible signal mediating phc quorum sensing in Ralstonia solanacearum. ChemBioChem 16:2309-2318.

Karamanos, Y. 2014. Purification and characterisation of lactate dehydrogenase: An undergraduate biochemistry laboratory experiment. Adv. Biochem. 2:14-23.

Kato, G. J., McGowan, V., Machado, R. F., Little, J. A., Taylor, J., 6th, Morris, C. R., Nichols, J. S., Wang, X., Poljakovic, M., Morris, S. M., Jr., and Gladwin, M. T. 2006. Lactate dehydrogenase as a biomarker of hemolysis-associated nitric oxide resistance, priapism, leg ulceration, pulmonary hypertension, and death in patients with sickle cell disease. Blood 107:2279-2285.

Kim, J. H., Bae, K. H., Byun, J. K., Lee, S., Kim, J. G., Lee, I. K., Jung, G. S., Lee, Y. M., and Park, K. G. 2017. Lactate dehydrogenase-A is indispensable for vascular smooth muscle cell proliferation and migration. Biochem. Biophys. Res. Commun. 492:41-47.

Kumar, J. S., Umesha, S., Prasad, K. S., and Niranjana, P. 2016. Detection of quorum sensing molecules and biofilm formation in Ralstonia solanacearum. Curr. Microbiol. 72:297-305.

Laouami, S., Messaoudi, K., Alberto, F., Clavel, T., and Duport, C. 2011 Lactate dehydrogenase A promotes communication between carbohydrate catabolism and virulence in Bacillus cereus. J. Bacteriol. 193: 1757-1766.

Leonard, S., Hommais, F., Nasser, W., and Reverchon, S. 2017. Plantphytopathogen interactions: Bacterial responses to environmental and plant stimuli. Environ. Microbiol. 19:1689-1716.

Lodi, T., O'Connor, D., Goffrini, P., and Ferrero, I. 1994. Carbon catabolite repression in Kluyveromyces lactis: Isolation and characterization of the KIDLD gene encoding the mitochondrial enzyme D-lactate ferricytochrome c oxidoreductase. Mol. Gen. Genet. 244:622-629.

Mansfield, J., Genin, S., Magori, S., Citovsky, V., Sriariyanum, M., Ronald, P., Dow, M., Verdier, V., Beer, S. V., Machado, M. A., Toth, I., Salmond, G., and Foster, G. D. 2012. Top 10 plant pathogenic bacteria in molecular plant pathology. Mol. Plant Pathol. 13:614-629.

Mat-Jan, F., Alam, K. Y., and Clark, D. P. 1989. Mutants of Escherichia coli deficient in the fermentative lactate dehydrogenase. J. Bacteriol. 171: 342-348.

Mercado-Lubo, R., Gauger, E. J., Leatham, M. P., Conway, T., and Cohen, P. S. 2008. A Salmonella enterica serovar typhimurium succinate dehydrogenase/fumarate reductase double mutant is avirulent and immunogenic in BALB/c mice. Infect. Immun. 76:1128-1134.

Milenkovic, D., Blaza, J. N., Larsson, N. G., and Hirst, J. 2017. The enigma of the respiratory chain supercomplex. Cell Metab. 25:765-776.

Miller, J. H. 1972. Experiments in Molecular Genetics. CSH Laboratory Press, Cold Spring Harbor, NY.

Pan, Y., Ye, T., and Gao, Z. 2017. Cloning and functional analysis of succinate dehydrogenase gene PSSDHA in Phytophthora sojae. Microb. Pathog. 108:40-48.

Park, S.-J., Tseng, C.-P., and Gunsalus, R. P. 1995. Regulation of succinate dehydrogenase ( $s d h C D A B)$ operon expression in Escherichia coli in response to carbon supply and anaerobiosis: Role of ArcA and Fnr. Mol. Microbiol. 15:473-482.

Parsek, M. R., Val, D. L., Hanzelka, B. L., Cronan, J. E., Jr., and Greenberg, E. P. 1999. Acyl homoserine-lactone quorum-sensing signal generation. Proc. Natl. Acad. Sci. U.S.A. 96:4360-4365. 
Passarella, S., Paventi, G., and Pizzuto, R. 2014. The mitochondrial L-lactate dehydrogenase affair. Front. Neurosci. 8:407.

Pecsi, I., Hards, K., Ekanayaka, N., Berney, M., Hartman, T., Jacobs, W. R., Jr., and Cook, G. M. 2014. Essentiality of succinate dehydrogenase in Mycobacterium smegmatis and its role in the generation of the membrane potential under hypoxia. MBio 5:e01093-e14.

Peyraud, R., Cottret, L., Marmiesse, L., Gouzy, J., and Genin, S. 2016. A resource allocation trade-off between virulence and proliferation drives metabolic versatility in the plant pathogen Ralstonia solanacearum. PLoS Pathog. 12:e1005939.

Sierotzki, H., and Scalliet, G. 2013. A review of current knowledge of resistance aspects for the next-generation succinate dehydrogenase inhibitor fungicides. Phytopathology 103:880-887.

Summermatter, S., Santos, G., Pérez-Schindler, J., and Handschin, C. 2013. Skeletal muscle PGC-1 $\alpha$ controls whole-body lactate homeostasis through estrogen-related receptor $\alpha$-dependent activation of $\mathrm{LDH} B$ and repression of LDH A. Proc. Natl. Acad. Sci. U.S.A. 110:8738-8743.

Sun, D., Zhuo, T., Hu, X., Fan, X., and Zou, H. 2017. Identification of a Pseudomonas putida as biocontrol agent for tomato bacterial wilt disease. Biol. Control 114:45-50.

Tamura, K., Peterson, D., Peterson, N., Stecher, G., Nei, M., and Kumar, S. 2011. MEGA5: Molecular evolutionary genetics analysis using maximum likelihood, evolutionary distance, and maximum parsimony methods. Mol. Biol. Evol. 28:2731-2739.

Tans-Kersten, J., Brown, D., and Allen, C. 2004. Swimming motility, a virulence trait of Ralstonia solanacearum, is regulated by FlhDC and the plant host environment. Mol. Plant-Microbe Interact 17:686-695.

Tans-Kersten, J., Huang, H., and Allen, C. 2001. Ralstonia solanacearum needs motility for invasive virulence on tomato. J. Bacteriol. 183: 3597-3605.

Thauer, R. K., Jungermann, K., and Decker, K. 1977. Energy conservation in chemotrophic anaerobic bacteria. Bacteriol. Rev. 41:100-180.

Tran, Q. M., Rothery, R. A., Maklashina, E., Cecchini, G., and Weiner, J. H. 2006. The quinone binding site in Escherichia coli succinate dehydrogenase is required for electron transfer to the heme b. J. Biol. Chem. 281:32310-32317.

Vandamme, P., and Coenye, T. 2004. Taxonomy of the genus Cupriavidus: A tale of lost and found. Int. J. Syst. Evol. Microbiol. 54:2285-2289.

von Bodman, S. B., Bauer, W. D., and Coplin, D. L. 2003. Quorum sensing in plant-pathogenic bacteria. Annu. Rev. Phytopathol. 41:455-482.

Yao, J., and Allen, C. 2006. Chemotaxis is required for virulence and competitive fitness of the bacterial wilt pathogen Ralstonia solanacearum. J. Bacteriol. 188:3697-3708.

Yao, J., and Allen, C. 2007. The plant pathogen Ralstonia solanacearum needs aerotaxis for normal biofilm formation and interactions with its tomato host. J. Bacteriol. 189:6415-6424.

Yao, T. T., Fang, S. W., Li, Z. S., Xiao, D. X., Cheng, J. L., Ying, H. Z., Du, Y. J., Zhao, J. H., and Dong, X. W. 2017. Discovery of novel succinate dehydrogenase inhibitors by the integration of in silico library design and pharmacophore mapping. J. Agric. Food Chem. 65:3204-3211.

Zhang, Y., Luo, F., Wu, D., Hikichi, Y., Kiba, A., Igarashi, Y., Ding, W., and Ohnishi, K. 2015. PrhN, a putative marR family transcriptional regulator, is involved in positive regulation of type III secretion system and full virulence of Ralstonia solanacearum. Front. Microbiol. 6:357.

Zhou, T., Qin, L., Zhu, X., Shen, W., Zou, J., Wang, Z., and Wei, Y. 2017 The D-lactate dehydrogenase MoDLD1 is essential for growth and infection-related development in Magnaporthe oryzae. Environ. Microbiol. 19:3938-3958.

Zuluaga, A. P., Puigvert, M., and Valls, M. 2013. Novel plant inputs influencing Ralstonia solanacearum during infection. Front. Microbiol. $4: 349$.

AUTHOR-RECOMMENDED INTERNET RESOURCES

RaptorX prediction portal: http://raptorx.uchicago.edu/accounts/login/?next=/myjobs

Berkeley Drosophila Genome Project promoter prediction tool: http://www.fruitfly.org/seq_tools/promoter.html 